\title{
Health action zones
}

\section{Offer the possibility of radical ideas which need rigorous evaluation}

$\mathrm{R}$ educing inequalities in health and moving away from the competitive market in health care are both important elements of government policy. Health action zones, mentioned in last month's white paper ${ }^{1}$ but announced beforehand, are one means of implementing these objectives. ${ }^{2}$ Is the health action zone initiative likely to succeed in its challenging aims of creating and sustaining partnerships to reduce inequalities and improve the clinical effectiveness and cost effectiveness of services?

Health action zones are intended to "bring together all those contributing to the health of the local population to develop and implement a locally agreed strategy for improving the health of local people." Health authorities and others are expected to bid jointly to run zones; no more than 10 bids will succeed, the schemes starting from April and planned to run for five to seven years. The guidance mentions resources only obliquely, though it does offer other possibilities for support. ${ }^{2}$ These include changing the rules for joint use of resources-for example, pooling resources across health and local authorities, and the private sector; and priority access to health service capital and the NHS strategic change special assistance fund.

As long ago as 1980 the Black reportproposed an experimental programme to provide a range of services in 10 areas of particularly high mortality and adverse social conditions. ${ }^{3}$ That was never implemented, but lookalike health action zones did appear in the 1980s as healthy cities initiatives, the Urban Programme, City Challenge, and single regeneration bids. Many challenge fund and single regeneration projects, targeted at communities with high deprivation, have, however, so far had only limited health objectives. Moreover, the elements missing from all these initiatives have been the ability to combine the resources of these different sorts of initiatives, to prevent dilution, and the lack of rigorous evaluation.

Increasing constraints on public sector funding, the legacy of competition within public sector services, and unproductive joint working relationships have resulted in fragmented services, mutual suspicion and a "Why should we?" rather than a "Why couldn't we?" culture. For a healthy action zone to work, it will need to build on the interagency successes that have been achieved despite the system, rather than because of it.

So what kinds of proposals can the Secretary of State expect? Bids are likely to fall into one of four interlinked categories. The first group might cover the deprived inner cities struggling to harness any support for dealing with their toughest problems such as severe mental illness, homelessness, and refugees' health. The second group might include maverick solutions which will test the government's willingness to allow new flexibilities within a health action zone-for example, pooling resources from housing, drugs, and social services for particular care groups. The third, or "low hanging fruit," group might build on local strengths and partnerships-perhaps in areas where there has been might also already have achieved coterminosity of health and local authority boundaries.

The fourth group might propose entirely new partnerships or issues and thus not be at risk of "collaboration fatigue." These might include reducing the incidence and morbidity from tuberculosis or asthma or developing more cost effective prevention and care for conditions such as cancers and HIV infection. Alternatively, the proposal might be to create a new public-private sector partnership to share the risk and provide new skills and resources for primary care development-for example, healthy living centres and primary care resource centres.

How should proposals be selected from such different types of initiatives, each with its own merits and risks? Firstly, if reducing inequalities in health is indeed the priority, then the Department of Health should follow the logic-but not the punitive approach-of the Department of Education, which is targeting its education action zones at deprived inner city areas where the education (and health) divide is most evident ${ }^{4}$ Proposals which aim to tackle the causes of ill health such as unemployment, education, and housing should be high on the agenda. Areas could easily, and fairly, be chosen in relation to explicit levels of deprivation and poor health in those populations. However, the department would also have to accept that inequalities do not disappear quickly. ${ }^{5}$ Such initiatives will probably not have much to show after seven years.

By contrast, more modest proposals may prove attractive because they might achieve benefits in a short period. Nor should the "maverick" group be ignored. These proposals will need careful assessment and support because of the risks of loosening financial and democratic accountability: unintended consequences may occur, but such proposals may genuinely result in a more cost effective use of resources.

Only time and evaluation will show whether the health action zone initiative is worth generalising. Projects should be evaluated for how they achieve change as well as for the health benefits they might produce. If the "commitment to share learning" in the guidance is serious, the approach must show that as much can be learnt from failures as from successes. This is more likely to help move us from the dark age of post-1974 joint planning to approaches to suit the next century.

Bobbie Jacobson Director of public health

East London and the City Health Authority, London E3 2AN (bobbie@elcha.co.uk)

Laurann Yen Fellow in organisational development Office of Public Management, London WC1X 8JT (office@opm.co.uk)

\footnotetext{
The new NHS: modern dependable. London: Stationery Office, 1997.

2 Department of Health. Health action zones: invitation to bid. London: Department of Health, 1997 (EL97(65).

Department of Health. Report of the working group on inequalities in health London: Department of Health, 1980.

Carvel J. Blunkett targets poor schools zones. Guardian 1997; 5 Dec:14

5 Drever F, Whitehead M. Health inequalities. London: Office for National Statistics, 1997.
} 